\title{
Effect of File Mobility Pattern on AODV, DSDV and ZRP Routing Protocols in MANET
}

\author{
Priyanshu Arya \\ M.Tech (WMC) Scholar \\ GRD-IMT, Dehradun
}

\author{
Sandip Vijay, Ph.D \\ HOD (ECE Deptt.) \\ DIT, Dehradun
}

\author{
Aarti Pandey \\ M.Tech (WMC) Scholar \\ GRD-IMT, Dehradun
}

\author{
Vishal Gupta, Ph.D \\ Director \\ GRD-IMT, Dehradun
}

\begin{abstract}
:
Ad-Hoc network is an infrastructure - less network which is created by deploying the mobile nodes carrying wireless devices, capable of transferring data between each other and network topology is dynamic and random in such a type of networks. As we know that routing protocols basically defines the set of rules to be adopted in finding the path between the nodes in the network to communicate with each other. This paper focuses on three routing protocols - AODV, ZRP, and DSDV of ad-hoc network. The main aim is to find the routing protocol giving best result at different source nodes having the file mobility pattern. The routing protocols under realistic mobility model provide higher Throughput, Packet Delivery Ratio and lower Normalized Routing Overhead \& Average Delay. The simulation platform used for evaluating the proposed approach is GloMoSim /Qual Net.
\end{abstract}

\section{Keywords}

AD-HOC networks, AODV, DSDV, ZRP, and File Mobility Pattern.

\section{INTRODUCTION}

Wireless networking is an emerging technology that allows various users to communicate information and services electronically regardless of their geographic position. Wireless networks can be classified in two types:

Infrastructure Networks (base station is fixed)

It consists of a network with fixed and wired gateways. A mobile host communicates with a bridge in the network (called base station) within its communication radius. The mobile unit can move geographically while communicating. When it goes out of range of one base station, it connects with new base station and starts communicating through it hence this technique are named as Handoff.

\section{Infrastructure less (Ad hoc) Networks}

In infrastructure less network all nodes are mobile and can be connected dynamically in an arbitrary manner. As the range of each node in wireless transmission is limited, so as to communicate with nodes outside the transmission range, a node needs to give the aid of its nearby hosts in forwarding packets to the destination. So all nodes of the network behave as routers and take part in discovery and maintenance of routes to other nodes in the network [1] [2]. $\mathrm{AD}-\mathrm{HOC}$ network is a self-organizing wireless network having mobile nodes with no fixed infrastructure. For providing communication throughout the entire network, nodes are designed to serve as relays if required which results in a distributed multi-hop network with a time-varying topology.
Because of infrastructure less and self-organizing property, ad-hoc network can be rapidly deployed to provide robust communication in a variety of hostile environments which makes ad hoc networks very appropriate for providing tactical communication for military, law enforcement, and emergency response efforts. It also plays a role in civilian forums, such as the electronic classroom, convention centres, and construction sites.
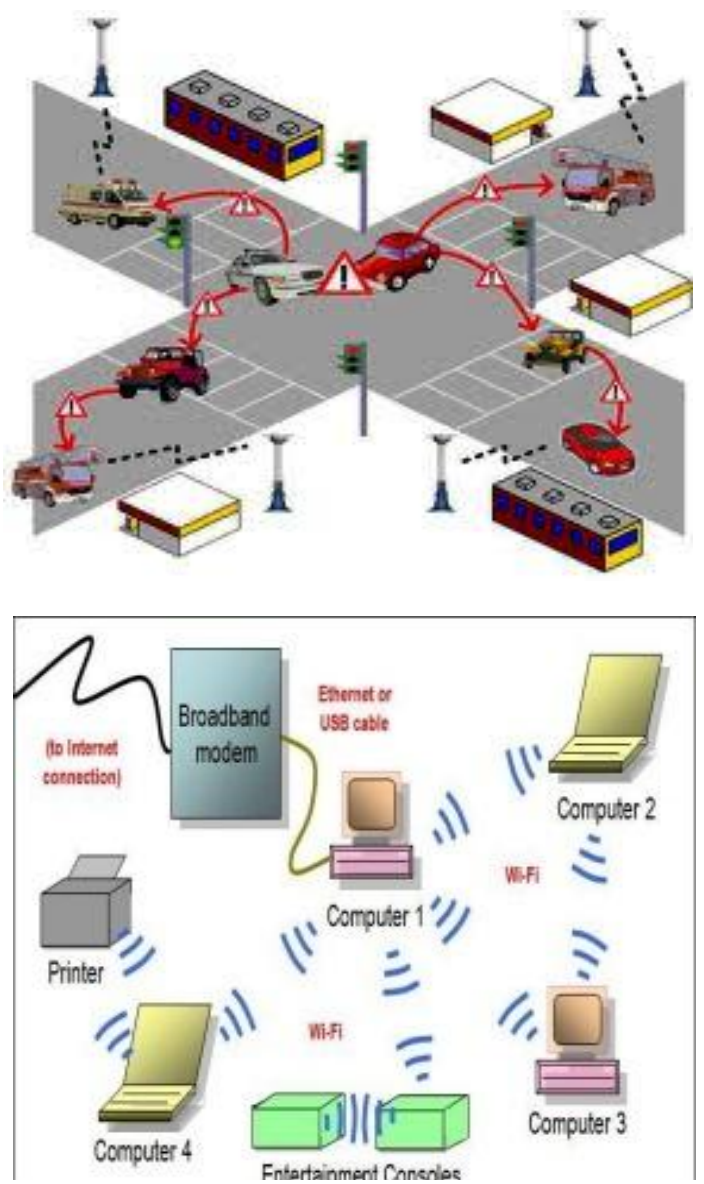

Fig.1 Example of an ADHOC Network.

In Ad - hoc network mobility of the nodes plays a very important role by affecting the number of an average connected path, which in turn affects the performance of the routing algorithm. 


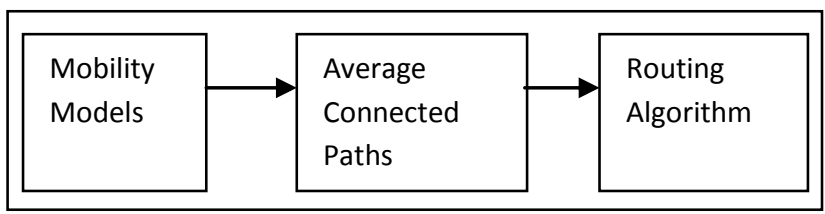

Fig.2 Connectivity path

\section{ROUTING PROTOCOLS DESCRIPTION IN AD -HOC NETWORK:}

The basic motive of a routing protocol is to find a path followed by the data packets from a source node to a destination node by specifying well defined set of rules. A variety of routing protocols for ad-hoc wireless network has been proposed in the recent past years. The routing protocol for ad-hoc wireless networks can be broadly classified into four categories:

\section{Routing information update mechanism \\ 2. Use of temporal information for routing \\ 3. Routing topology \\ 4. Utilization of specific resources}

The routing protocol discussed here belongs to the first category [3].

Table-Driven Routing Protocols: In table driven routing protocols, consistent and up-to-date routing information to all nodes is maintained at each node. These protocols are usually termed as proactive protocols as they maintain the routing information even before it is needed [4].

On-Demand Routing Protocols: In On-Demand routing protocols, the routes are created on the requirement of the users. If a user wants to send a packet to another node then this protocol searches for the route in an on-demand manner and establishes the connection in order to transmit and receive the packet [5] from one end to other end.

Hybrid Routing Protocols: It combine the best features of the above two categories. Nodes within a certain distance from the node concerned, or within a particular geographical region, are said to be within the routing zone of the given nodes. For routing within this zone, a table-driven approach is used. For nodes that are beyond this zone, an on- demand technique is used.

\section{Destination Sequenced Distance Vector (DSDV) Protocol:}

The destination sequenced distance vector routing protocol is a proactive routing protocol which is a modification of conventional Bellman-Ford routing algorithm. This protocol adds a new attribute, sequence number, to each route table entry at each node. Routing table is maintained at each node and with these table nodes able to transmit the packets from one node to other nodes in the network. The sequence number is used to distinguish stale routes from new ones and thus avoid the formation of loops. The stations periodically generate and transmit their routing tables to their immediate neighbors. A station also transmits its routing table if a significant change has occurred in its table from the last update sent. So the update is both time-driven and eventdriven. When the network is relatively stable, incremental updates are sent to avoid extra traffic and full dump are relatively infrequent. In a fast-changing network, incremental packets can grow big so full dumps will be more frequent.
DSDV protocol guarantees loop free paths and Count to infinity problem is reduced in DSDV [6].On the contrary in DSDV there is wastage of bandwidth due to unnecessary advertising of routing information even if there is no change in the network topology [7] also DSDV doesn't support Multi path Routing. It is difficult to determine a time delay for the advertisement of routes [8].

\section{Ad-hoc On-Demand Distance Vector (AODV) Protocol:}

AODV is a very simple, efficient, and effective routing protocol for Mobile Ad-hoc Networks which again do not have fixed topology. This algorithm was motivated by the limited bandwidth that is available in the media that are used for wireless communications. The on demand route discovery and route maintenance from DSR and hop-by-hop routing, usage of node sequence numbers from DSDV make the algorithm cope up with topology and routing information. Establishing the routes purely on-demand makes AODV a very useful and desired algorithm for MANETs [1]. AODV [3] discovers routes as needed by the user. However, AODV obeys very different mechanism to maintain routing information. It uses traditional routing tables, one entry per destination. This is in contrast to DSR, which can maintain multiple route cache entries for each destination. Without source routing, AODV relies on routing table entries to propagate an RREP back to the source and, subsequently, to route data packets to the destination. AODV uses sequence numbers maintained at each destination to determine freshness of routing information and to prevent routing loops.

\section{Zone Routing Protocol (ZRP):}

Hybrid routing combines characteristics of both reactive and proactive routing protocols to make routing more scalable and efficient [9]. Mostly hybrid routing protocols are zone based; it means the number of nodes is divided into different zones to make route discovery and maintenance more reliable for MANET. The need of these protocols arises with the deficiencies of proactive and reactive routing and there is a demand of such a protocol that can resolve the problem of both. ZRP limits the range of proactive routing methods to neighboring nodes locally; however ZRP uses reactive routing to search the desired nodes by querying the selective network nodes globally instead of sending the query to all the nodes in network. ZRP uses "Intrazone" and "Interzone" routing to provide flexible route discovery and route maintenance in the multiple ad hoc environments. Interzone routing performs route discovery through reactive routing protocol globally while intrazone routing based on proactive routing in order to maintain up-to-date route information locally within its own routing range. One of the important characteristic of ZRP is that it reduces the network overhead that is caused by proactive routing and it also handles the network delay that is caused by reactive routing protocols and perform route discovery more efficiently.

\section{MOBILITY MODELS IN WIRELESS AD-HOC NETWORK:}

The mobility model is basically designed to describe the movement pattern of mobile nodes, and how their location, velocity and acceleration changes over time. As mobility patterns play a significant role in determining the protocol performance, it is desirable for mobility models to emulate the movement pattern of targeted real life applications in a 
reasonable way. Mobility models are used for simulation purposes when new network protocols are evaluated.

\section{File Mobility Model:}

The nodes move according to a file specifying node movement at different simulation times. The file contains waypoints for each node specifying node's next position, time at which the node arrives at the location and orientation (optionally). The node moves from one position to the next in a straight line at a constant speed as shown in Figure-3.

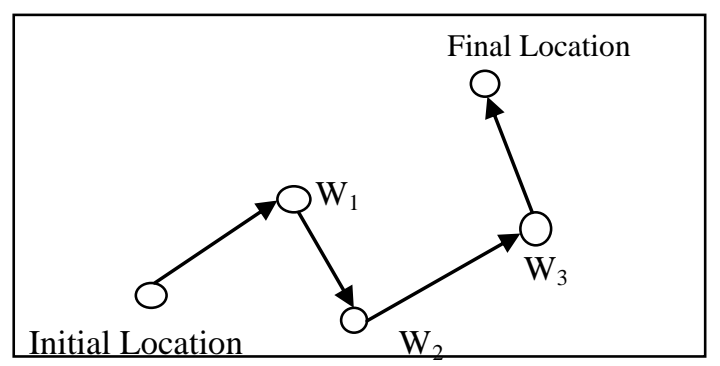

Fig.3 File mobility model

\section{SIMULATION TOOL:}

The simulation platform used for evaluating the proposed approach is GloMoSim (Lokesh Bajaj et al, 1999), a discrete event detailed simulator for wireless network systems. It is based on C-based parallel simulation language PARSEC (R. Bagrodia et al, 1998). The objective of IEEE 802.11 standard is to provide wireless connectivity to wireless devices/ nodes that require rapid deployment, which may be portable, or which may be mounted on moving vehicles within local area. The IEEE 802.11 also aids the regulatory bodies in standardizing access to one or more radio frequency bands for the purpose of local area communication. The interfaces offered by 802.11 to the higher layers are the same as those offered in other 802.x standards. Qual Net enables users to Design a new protocol models, Optimize new and existing models, Design large wired and wireless networks using preconfigured or user-designed models, Analyze the performance of networks and perform what-if analysis to optimize them. Qual Net is the preferable simulator for the ease of operation.

\section{PERFORMANCE METRICS: \\ THROUGHPUT:}

It is defined as the fraction of the channel capacity used for useful transmission selects a destination at the beginning of the simulation i.e., information whether or not data packets correctly delivered to the destinations [10].

\section{Average end to end delay:}

The average end-to-end delay of data packets is the interval between the data packet generation time and the time when the last bit arrives at the destination. This parameter basically deals with the network speed and communication effectiveness. Higher the delay, lower is the speed and possibility of packet drop and so needs the fault tolerance approach of selecting these protocols [10].

\section{Average routing overhead (ARH):}

Average routing overhead is the total number of routing packets divided by total number of delivered data packets [13].
$\mathrm{ARH}=$ Total no of routing packets/Total no of delivered data packets.

\section{Average Jitter:}

The jitter is the variation of data communication packets in the network [11]. It is the variation in the time between packets arriving, caused by network congestion, timing drift, or route changes [12].

\section{Packet Delivery Ratio:}

Packet Delivery Ratio (PDR): The number of data packets sent from the source to the number of received at the destination [13].

PDR $=($ control packets sent-delivery packet sent $)$ control packets sent.

Table 1: Parametric Table

\begin{tabular}{|l|c|}
\hline \multicolumn{1}{|c|}{ PARAMETRS } & VALUE \\
\hline $\begin{array}{l}\text { No. Of Source } \\
\text { Modes }\end{array}$ & $4,6,8,10,14$ \\
\hline Mobility Models & File Mobility Model \\
\hline Routing Protocols & $\begin{array}{c}\text { AOLLM , } \\
\text { and ZRP }\end{array}$ \\
\hline Pause Time & 30 sec \\
\hline Node Density & CBR \\
\hline $\begin{array}{l}\text { Data Traffic } \\
\text { Pattern }\end{array}$ & 30 Sec \\
\hline Simulation Time & $1500 * 1500$ \\
\hline Terrain & 0 - 30 Sec \\
\hline Speed & 1 packet/sec \\
\hline CBR Traffic Rate & 512 bytes \\
\hline Packet Size & 802.11 \\
\hline MAC Layer & \\
\hline
\end{tabular}

\section{RESULT AND DISCUSSION: THROUGHPUT}

The nodes move according to a file specifying node movement at different simulation times. As the number of sources increasing means as the traffic increases the throughput is high when there are 14 number of source nodes in the case of AODV protocol as compared to other to protocols. This is because here the speeds of the mobile nodes are constant. 
Table 2: Throughput table for different number of sources

\begin{tabular}{|l|c|c|c|c|c|}
\hline \multicolumn{1}{|c|}{$\begin{array}{c}\text { No of source } \\
\text { nodes }\end{array}$} & 4 & 6 & 8 & 10 & 14 \\
\hline AODV (FM) & 15071 & 21044 & 27082 & 32697 & 41621 \\
\hline $\begin{array}{l}\text { BELLMENFORD } \\
\text { (FM) }\end{array}$ & 10342 & 12278 & 16980 & 21965 & 27986 \\
\hline ZRP (FM) & 10512 & 13464 & 14682 & 17000 & 31612 \\
\hline
\end{tabular}

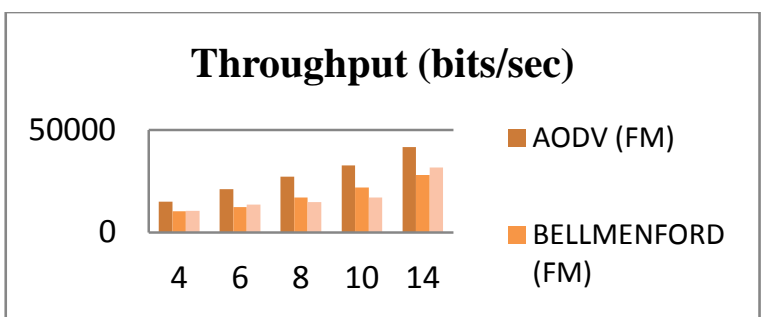

Fig.4 Throughput bar graph for different number of sources

Average End To End Delay: Here I observed that as the number of source nodes increasing there is an increase in the average end to end delay for all the cases of protocols. Also it is noticed that there is a slight change in AODV and Bellman Ford protocol as both have minimum number of average end to end delay at less number of source nodes.

Table 3: AEED table for different number of source

\begin{tabular}{|l|l|l|l|l|l|}
\hline $\begin{array}{l}\text { No of } \\
\text { source } \\
\text { nodes }\end{array}$ & & \multicolumn{1}{|c|}{$\mathbf{4}$} & \multicolumn{1}{|c|}{$\mathbf{6}$} & \multicolumn{1}{|c|}{$\mathbf{1 0}$} & \multicolumn{1}{|c|}{$\mathbf{1 4}$} \\
\hline & 0.179 & 0.524 & 0.745 & 1.322 & 2.687 \\
AODV (FM) & 255 & 83 & 357 & 95 & 29 \\
\hline BELLMENF & 0.157 & 0.295 & 0.337 & 0.808 & 1.202 \\
ORD (FM) & 494 & 813 & 788 & 129 & 65 \\
\hline & 0.406 & 0.598 & 1.400 & 1.668 & 2.096 \\
ZRP (FM) & 096 & 28 & 49 & 67 & 71 \\
\hline
\end{tabular}

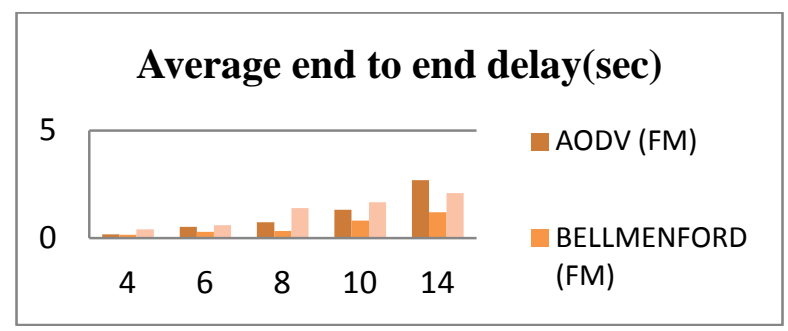

Fig.5 AEED bar graph for different number of sources

Average Routing Overhead: In this there is approximately linear variation in AODV and bellman ford routing protocol and minimum amount of over head is noticed in ZRP and bellman ford routing protocol.

Table 4: Overhead table for different number of sources

\begin{tabular}{|l|l|l|l|l|l|}
\hline $\begin{array}{c}\text { No of source } \\
\text { nodes }\end{array}$ & \multicolumn{1}{|c|}{$\mathbf{4}$} & \multicolumn{1}{|c|}{$\mathbf{6}$} & \multicolumn{1}{|c|}{$\mathbf{8}$} & \multicolumn{1}{|c|}{$\mathbf{1 0}$} & \multicolumn{1}{|c|}{$\mathbf{1 4}$} \\
\hline & 3.65 & 5.0689 & 6.5517 & 7.862 & 9.965 \\
AODV (FM) & 51 & 6 & 2 & 069 & 5 \\
\hline BELLMENF & 2.51 & 2.9310 & 4.1379 & 5.351 & 6.655 \\
ORD (FM) & 72 & 345 & 31 & 034 & 1 \\
\hline & 2.51 & 3.2068 & 3.3793 & 3.689 & 7.379 \\
ZRP (FM) & 72 & 9 & 103 & 6 & 31 \\
\hline
\end{tabular}

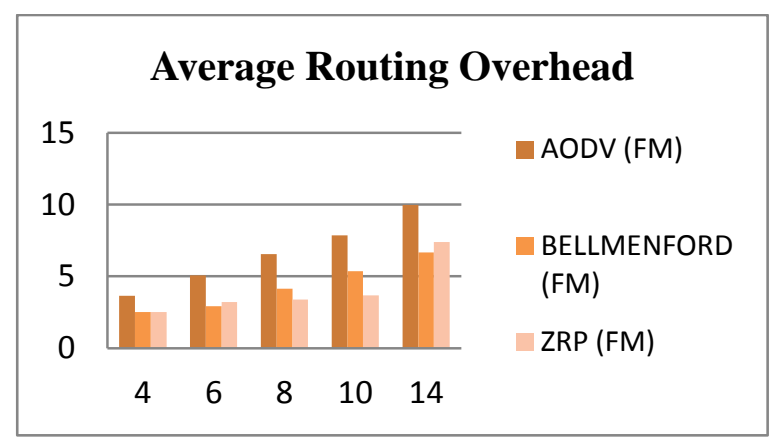

Fig.6 Overhead bar graph for different number of sources

Average Jitter: The result can be so analyze that as the minimum value of average jitter can be seen for the case of AODV and ZRP also for both the case there is not a linear variation in values. But the maximum and minimum variation is for case of ZRP routing protocol thus it will work better for this scenario.

Table 5: Jitter table for different number of sources

\begin{tabular}{|c|c|c|c|c|c|}
\hline $\begin{array}{ll}\text { No } & \text { of } \\
\text { source } & \\
\text { nodes } & \end{array}$ & 4 & $\rightarrow$ & 8 & 10 & 14 \\
\hline $\begin{array}{l}\text { AODV } \\
\text { (FM) }\end{array}$ & $\begin{array}{l}0.0586 \\
545\end{array}$ & $\begin{array}{l}0.1761 \\
03\end{array}$ & $\begin{array}{l}0.258 \\
699\end{array}$ & $\begin{array}{l}0.328 \\
912\end{array}$ & $\begin{array}{l}0.748 \\
32\end{array}$ \\
\hline BELLMEN & 0.4230 & 0.6308 & 0.107 & 0.148 & $\begin{array}{l}0.223 \\
896\end{array}$ \\
\hline ZRP (FM) & $\begin{array}{l}0.0757 \\
46\end{array}$ & $\begin{array}{l}0.0668 \\
519\end{array}$ & $\begin{array}{l}0.126 \\
731\end{array}$ & 0.1 & $\begin{array}{l}0.209 \\
013\end{array}$ \\
\hline
\end{tabular}

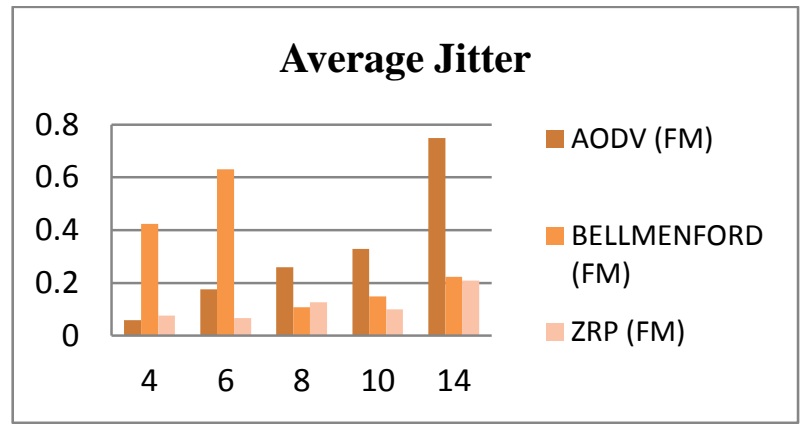

Fig.7 Average Jitter Bar graph for different number of sources

Packet Delivery Ratio: In this scenario file mobility model is applied to the network here also same thing is noted that AODV routing protocol works best as compared to other to protocol with the highest value of packet delivery ratio hence minimum amount of packet drops is noticed. 
Table 6: PDR table for different number of sources

\begin{tabular}{|l|l|l|l|l|l|}
\hline $\begin{array}{l}\text { No of } \\
\text { source } \\
\text { nodes }\end{array}$ & \multicolumn{1}{|c|}{$\mathbf{4}$} & \multicolumn{1}{|c|}{$\mathbf{6}$} & \multicolumn{1}{|c|}{$\mathbf{8}$} & \multicolumn{1}{|c|}{$\mathbf{1 0}$} & \multicolumn{1}{c|}{$\mathbf{1 4}$} \\
\hline & 365. & 506.89 & 655.17 & 786.2 & 996.5 \\
AODV (FM) & 51 & 6 & 2 & 069 & 5 \\
\hline BELLMENF & 251. & 293.10 & 413.79 & 535.1 & 665.5 \\
ORD (FM) & 72 & 345 & 31 & 034 & 1 \\
\hline & 251. & 320.68 & 337.93 & 368.9 & 737.9 \\
ZRP (FM) & 72 & 9 & 103 & 6 & 31 \\
\hline
\end{tabular}

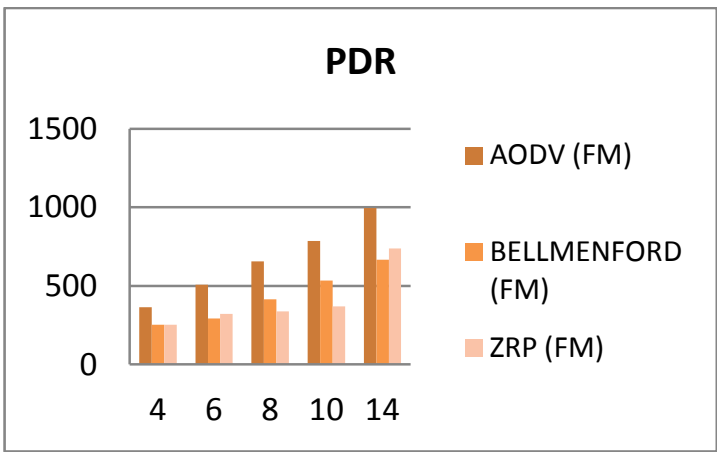

Fig.8 PDR bar graph for different number of source

\section{CONCLUSION:}

After performing the experiment I able to conclude that, AODV routing protocols shows higher value of Average Jitter than all the other two routing protocol used in the experiment as the number of source nodes are increasing hence the variation in the data communication packets is high and in turn the possibility of congestion will also more. For the case of AEED I have noted that it is quite high for AODV and very less for DSDV and ZRP and same thing is noted for the case of Average routing overhead and because of which packets in AODV travel at lower speed and in turn the number of delivered data packet are low thus packet drop will also be high enough but it will low for the case of DSDV and ZRP. AODV shows highest value of packet delivery ratio followed by DSDV and ZRP routing protocols.

\section{REFERENCES:}

[1] Dr. Aditya Goel \& Ajaii Sharma , "Performance Analysis of Mobile Ad-hoc Network Using AODV Protocol", International Journal of Computer Science and Security (IJCSS), Volume (3): Issue (5), 2007 pp.334343.

[2] Bhavyesh Divecha, Ajith Abraham, Crina Grosan, Sugata Sanyal, "Impact of Node Mobility on MANET Routing Protocols Models", Journal of Digital Information Management, Volume 5 Number 1 , February 2007,pp.19-24.

[3] E. Royer and C. Toh, "A Review of Current Routing Protocols for Ad-Hoc Mobile Wireless Networks", IEEE Personal Communications, Vol. 6, No. 2, Apr 1999, pp. 46-55.

[4] Sung-Ju Lee, William Su, and Mario Gerla, "Wireless ad-hoc multicast routing with mobility prediction" ACM/ Kluwer Mobile Networks and Applications, special issue on Design and Deployment of Ad Hoc Networks, vol. 6, no. 4, August 2001, pp. 351-360.

[5] Sung-Ju Lee, Elizabeth M. Belding-Royer, and Charles E. Perkins, "Ad hoc On-Demand Distance-Vector Routing Scalability", ACM Mobile Computing and Communications Review, vol. 6, no. 3, July 2002, pp. 94-95.

[6] Yi Lu, Weichao Wang, Yuhui Zhong, Bharat Bhargava, "Study of Distance Vector Routing Protocols for Mobile Ad Hoc Networks", Proceedings of the First IEEE International Conference on Pervasive Computing and Communications (PerCom'03), Mar 2003, pp. 187-194.

[7] Peter Baumung, Martina Zitterbart and Kendy Kutzner "Improving Delivery Ratios for Application Layer Multicast in Mobile Ad-hoc Networks", journal of computer communication,vol.28, Issue 14,sept 2005,pp.1669-1679.

[8] D. Bein, A. K. Datta, C. R. Jagganagari and V. Villain, "A Self-stabilizing Link- Cluster Algorithm in Mobile Ad Hoc Networks", Proceedings of The International Symposium on Parallel Architectures, Algorithms, and Networks (I-SPAN)vol.4,no.2, Dec 2005, pp. 436-441.

[9] M.L Sharma, Noor Fatima Rizvi , Nipun Sharma ,Anu Malhan and Swati Sharm, "Performance Evaluation of MANET Routing Protocols under CBR and FTP traffic classes”, Int. J. Comp. Tech. Appl., vol. 2 (3),2010, 392 400 .

[10] Dr.V.B.Narsimha1* B.Manjula B.Sujatha K.Arun Raj Bapuji and S.Nagaprasad, "Comparison of multicast routing protocol in $\mathrm{AD}-\mathrm{HOC}$ wireless network", International Journal on Computer Science and Engineering (IJCSE), Vol. 3 No. 2 Feb 2011, pp. 871876.

[11] 1411 Rachit Jain, Laxmi Shrivastava ,"Study and Performance Comparison of AODV \& DSR on the basis of Path Loss Propagation Models", International Journal of Advanced Science and Technology Vol. 32, July 2011,pp.45-52.

[12] Humayun Bakht has published his paper on "Survey of Routing Protocols for Mobile Ad-hoc Network", In International Journal of Information and Communication Technology Research Volume 1 No. 6, October 2011, pp. 258-270.

[13] Tracy Camp, Jeff Boleng and Vanessa Davies ,"A Survey of Mobility Models for Ad Hoc Network Research", Wireless Communication \& Mobile Computing (WCMC): Special issue on Mobile Ad Hoc Networking Research, Trends and Applications, vol. 2, no. 5, pp. 483-502, 2002

[14] C. Bettstetter, G. Resta, and P. Santi, "The node distribution of the random waypoint mobility model for wireless ad hoc networks", IEEE Trans. Mobile Comput., 2(3), July - September 2003,pp. 257-269.

[15] J. Yoon, M. Liu and B. Noble, "Random Waypoint Considered Harmful", in Proceedings of INFOCOM, IEEE, Vol. 2, Apr 2003, pp. 1312-1321. 